Chronic Obstructive Pulmonary Diseases:

Journal of the COPD Foundation

Original Research

\title{
Evaluation of Systemic Corticosteroids in Patients With an Acute Exacerbation of COPD and a Diagnosis of Pneumonia
}

Tyler Scholl, PharmD ${ }^{1}$ Tyree H. Kiser, PharmD ${ }^{1}$ Sheryl F. Vondracek, PharmD ${ }^{1}$

\section{Abstract}

Background: Chronic obstructive pulmonary disease (COPD) and pneumonia are leading causes of morbidity and mortality and are frequently comorbid. Studies of systemic corticosteroids in pneumonia have shown conflicting outcomes, whereas studies in acute exacerbations of COPD (AECOPD) have shown significant benefits. No studies have evaluated systemic corticosteroids in patients with both an AECOPD and pneumonia.

Purpose: To evaluate the use of systemic corticosteroids in patients with both an AECOPD and pneumonia.

Patients and Methods: Patients with a diagnosis of both COPD or obstructive chronic bronchitis with exacerbation and pneumonia admitted to the University of Colorado Hospital between July 1, 2012 and May 20, 2016 were retrospectively evaluated. Patients who received systemic corticosteroids were compared to those that did not. The primary outcome was length of hospital stay (LOHS). Secondary outcomes were in-hospital treatment failure, a composite of intensive care unit (ICU) admission, ventilation, and escalation of steroid therapy, 30-day AECOPD or pneumonia readmission, and 30-day mortality.

Results: A total of 138 patients were included- 89 in the steroid group and 49 in the non-steroid group. No significant differences in baseline characteristic were noted. No difference was seen in mean LOHS (4.7 \pm 3.2 versus $4.2 \pm 2.1$ days, $p=0.27$ ), in-hospital treatment failure ( $7 \%$ versus $4 \%, p=0.72$ ), 30 -day readmission or 30 -day mortality between the steroid and non-steroid groups, respectively. There was a difference in mean LOHS for patients with severe pneumonia between the steroid and non-steroid groups ( $6.0 \pm 4.0$ versus $4.3 \pm 1.8 ; p=0.03$ ).

Conclusions: This study suggests that systemic corticosteroids may not provide a clinical benefit to patients with an AECOPD and pneumonia.

Abbreviations: chronic obstructive pulmonary disease, COPD; acute exacerbation of chronic obstructive pulmonary disease, AECOPD; intensive care unit, ICU; length of hospital stay, LHOS; community-acquired pneumonia, CAP; International Classification of Diseases-9th Revision, ICD-9; pneumonia severity index, PSI; inhaled corticosteroids, ICS; long-acting beta2-agonist, LABA; long-acting muscarinic antagonist, LAMA; Veteran's Affairs, VA

Funding Support: not applicable

Date of Acceptance: October 24, 2017

Citation: Scholl T, Kiser TH, Vondracek SF. Evaluation of systemic corticosteroids in patients with an acute exacerbation of COPD and a diagnosis of pneumonia. Chronic Obstr Pulm Dis. 2018;5(1):57-65. doi: https://doi.org/10.15326/jcopdf.5.1.2017.0157

1 University of Colorado Skaggs School of Pharmacy and Pharmaceutical Sciences, Aurora

\section{Keywords:}

hospitalized; length of stay; pneumonia; COPD; exacerbation; corticosteroids

\section{Address correspondence to:}

Sheryl F. Vondracek, PharmD

University of Colorado

Skaggs School of Pharmacy and Pharmaceutical Sciences

12850 E. Montview Blvd. C238

Aurora, CO 80045

Phone: (303) 7242638

Email: sheryl.vondracek@ucdenver.edu 


\section{Introduction}

Chronic obstructive pulmonary disease (COPD) is a leading cause of morbidity and mortality worldwide. In 2014 , it was the third leading cause of death in the in United States, behind only heart disease and cancer. ${ }^{1}$ Globally, it is the 4th leading cause of death with an estimated 3 million deaths annually. ${ }^{2}$ Exacerbations account for most of the morbidity, mortality, and costs associated with COPD and infections play an important role in their etiology. ${ }^{3,4}$ Pneumonia is the leading cause of death from infectious disease in the United States. In 2014, it was the eighth overall leading cause of death in the United States, causing 50,622 deaths. ${ }^{1,5}$ Patients with COPD are at increased risk of pneumonia because of impaired lung defenses and possibly inhaled corticosteroid use. ${ }^{6,7}$ A population-based cohort study in Denmark showed that approximately $36 \%$ of patients hospitalized for a first-time COPD exacerbation also received a pneumonia diagnosis. ${ }^{8}$ Patients hospitalized for COPD exacerbations and pneumonia have worse outcomes with increased health care utilization, longer length of hospital stay, and a higher mortality. ${ }^{8}$

Systemic corticosteroids are standard of care in the management of an acute exacerbation of chronic obstructive pulmonary disease (AECOPD). ${ }^{9}$ Studies have shown that their use improves short-term lung function and reduces treatment failure rates, 30-day relapse, and length of hospital stay. ${ }^{9,10}$ However, studies examining the effectiveness of systemic corticosteroid therapy in an AECOPD have universally excluded patients with pneumonia. ${ }^{10,11}$ The use of corticosteroids in community-acquired pneumonia (CAP) has been controversial with some studies showing a faster improvement in clinical stability, shorter length of hospital stay, and decreased mortality, while others have shown no benefit with an increase in side effects (especially hyperglycemia). ${ }^{12,13}$ Studies examining the use of systemic corticosteroids in patients with CAP have included only small numbers of patients with COPD. ${ }^{14}$ Therefore, the benefits of systemic corticosteroid use in patients with an AECOPD and CAP remains unknown.

The purpose of this study was to examine the use of systemic corticosteroids in patients with an AECOPD and CAP. We hypothesized that there would be no statistically significant difference in length of hospital stay between patients with an AECOPD and CAP who received a course of systemic corticosteroids and those who did not.

\section{Methods}

\section{Study Design}

This was a retrospective evaluation of patients 40-89 years of age admitted to the University of Colorado Hospital (a 648-bed urban academic medical center) between July 1, 2012 and May 20, 2016 with an AECOPD and CAP. Patients during this period with a diagnosis of either COPD (International Classification of Diseases-9th Revision [ICD-9] code 496, ICD-10th Revision [ICD-10] code J44.9) or obstructive chronic bronchitis with exacerbation (ICD-9 code 491.21, ICD-10 code J44.1) on their hospital diagnosis list and an admission diagnosis of pneumonia (ICD-9 code 486, ICD-10 code J18.9) were examined for inclusion. Patients were excluded if they had a diagnosis of hospital-acquired pneumonia, no radiographic evidence of pneumonia, no evidence of an AECOPD, an immunocompromised condition (human immunodeficiency virus, solid organ or bone marrow transplant, or receiving immunosuppressive therapy), asthma or other lung disease, developed septic shock with the need for vasopressors, received oral or IV corticosteroids within 30 days of admission, or died during hospitalization. All data were extracted from the electronic health record, EPIC (Madison, Wisconsin). The Colorado Multiple Institutional Review Board approved this study.

Patients who received a treatment course of systemic corticosteroids (oral prednisone or IV methylprednisolone) during their hospital stay were compared to patients who did not receive systemic corticosteroids. To be included in the steroid group, patients received systemic corticosteroids for more than 1 dose, or for 1 dose with a discharge course of systemic corticosteroids immediately thereafter. All steroid dosing was converted to prednisone equivalents. ${ }^{15}$

\section{Outcomes}

The primary outcome was length of hospital stay. Our main secondary outcome was in-hospital treatment failure, which was defined as intensive care unit (ICU) admission or mechanical or non-invasive ventilation as a result of treatment failure, or escalation of steroid therapy. Patients were only included up to once for this outcome, even if they met more than one criteria for it. We also assessed each of these components individually for both groups. ICU admission and 
ventilation were attributed to treatment failure if they occurred 6 hours or more after the first dose of steroid (steroid group) or 6 hours or more after hospital admission (non-steroid group). Other secondary outcomes included 30-day AECOPD or pneumonia readmission and 30-day mortality in patients with evaluable data. In addition to comparing outcomes in the steroid and non-steroid groups, we also performed a subgroup analysis on patients admitted with severe pneumonia, i.e., having a pneumonia severity index (PSI) score of IV or V.

Common adverse events caused by systemic corticosteroid were evaluated. To assess for hyperglycemia, we recorded the frequency of insulin administration in patients without a diagnosis of diabetes and the frequency of hyperglycemia (serum glucose $>200 \mathrm{mg} / \mathrm{dL}$ ) requiring additional insulin (lispro) therapy in patients with a diagnosis of diabetes. To assess for psychiatric adverse effects (acute psychosis, agitation, anxiety, or insomnia) we recorded the frequency of a new order for an antipsychotic or anxiolytic medication for psychosis, agitation, or anxiety and the frequency of a new order for a sedative/hypnotic agent for sleep. To assess for edema, we recorded the frequency of a new order for a loop diuretic to treat edema.

\section{Statistical Analysis}

For comparisons between the 2 groups, the Fisher's exact test was used for categorical variables and continuous variables were evaluated using a student t-test for non-matched samples with unequal variance. All tests were 2 -tailed and a $p$-value $<0.05$ was considered significant. All continuous variables are reported as a meantstandard deviation. Based on previous studies, we estimated that approximately 64 patients would be needed in each group to have $80 \%$ power to detect a 2-day difference in length of stay assuming a population mean length of stay of 8 days, a standard deviation of 4 days, and an alpha level of 0.05. ${ }^{10,12,16}$ All statistical calculations were performed using Graph Pad 2016 (San Diego, California).

\section{Results}

A total of 138 patients were enrolled in this study based on inclusion and exclusion criteria. Eightynine (64\%) were included in the steroid group, and $49(36 \%)$ in the non-steroid group. The patients in the steroid and non-steroid groups were similar with no significant differences in key characteristics (Table1). The steroid group received a mean daily dose of $65.3 \pm 86.3 \mathrm{mg}$ prednisone equivalents. Twenty-eight percent of patients received at least 1 dose of IV methylprednisolone. The mean total duration of steroid therapy was $7 \pm 4.7$ days with $82 \%$ of patients receiving $\leq 7$ days total. The percentage of patients with a guideline recommended initial antibiotic regimen for CAP was similar between the 2 groups with 74\% in the steroid group and $80 \%$ in the non-steroid group receiving the combination of a beta-lactam or vancomycin plus azithromycin or doxycycline or levofloxacin alone $(p=0.54) .{ }^{17}$ Fiftyone percent of patients in the steroid group and $48 \%$ in the non-steroid group $(p=0.10)$ received an inhaled corticosteroid (ICS) prior to admission. One or more maintenance COPD meds were continued during hospitalization in $58.4 \%$ of patients in the steroid group and $44.9 \%$ of patients in the non-steroids group $(p=0.15)$.

There was no difference in the primary outcome with the steroid group having a mean length of stay of $4.7 \pm 3.2$ day and the non-steroid group of $4.2 \pm 2.1$ days $(p=0.27)$. There was also no difference in the secondary outcomes of in-hospital treatment failure, 30-day readmission for an AECOPD or pneumonia or 30-day mortality between the steroid and non-steroid groups (Table 2). Although we observed more hyperglycemia in the steroid group, there was no difference between groups in any of the adverse events (Table 3 ).

Among patients with severe pneumonia, there was no difference in mean age or mean PSI score between the steroid and non-steroid groups. There was also no difference in the frequency of in-hospital treatment failure, 30-day readmission or 30-day mortality. However, there was a significantly longer length of hospital stay in the steroid group than in the nonsteroid group ( $6.0 \pm 4.0$ versus $4.3 \pm 1.8$ days, $p=0.03$ ) (Table 4). 


\section{Table 1. Baseline Characteristics of the Study Population}

\begin{tabular}{|c|c|c|c|}
\hline & $\begin{array}{l}\text { Steroid Group } \\
\qquad(n=89)\end{array}$ & $\begin{array}{l}\text { Non-Steroid } \\
\text { Group }(n=49)\end{array}$ & $p$-value \\
\hline Age (Years), mean $\pm \mathrm{SD}$ & $67.5 \pm 11.5$ & $67.9 \pm 12.4$ & 0.85 \\
\hline Male, n (\%) & $50(56 \%)$ & $29(59 \%)$ & 0.86 \\
\hline \multicolumn{4}{|l|}{ Race } \\
\hline White, $\mathrm{n}(\%)$ & 67 (75\%) & 35 (71\%) & 0.69 \\
\hline Black or African-American, $\mathrm{n}(\%)$ & $7(8 \%)$ & $4(8 \%)$ & 0.99 \\
\hline \multicolumn{4}{|l|}{ Smoking Status } \\
\hline Current Smoker, n (\%) & $34(38 \%)$ & $16(33 \%)$ & 0.58 \\
\hline Past Smoker, n (\%) & $54(61 \%)$ & $29(59 \%)$ & 0.99 \\
\hline Never Smoker, n (\%) & $0(0 \%)$ & $2(4 \%)$ & 0.12 \\
\hline Pack Years of Smoking, mean \pm SD & $38.9 \pm 28.8$ & $33.2 \pm 27.3$ & 0.35 \\
\hline \multicolumn{4}{|l|}{ Clinical Severity } \\
\hline PSI Score at Admission, mean \pm SD & $85.8 \pm 26.1$ & $88.7 \pm 25.4$ & 0.52 \\
\hline Severe Pneumonia (PSI Score IV or V), n (\%) & $37(42 \%)$ & $25(51 \%)$ & 0.37 \\
\hline Direct Admission to ICU & $21(24 \%)$ & $5(10 \%)$ & 0.07 \\
\hline Bacteremia & $5(6 \%)$ & $1(2 \%)$ & 0.42 \\
\hline \multicolumn{4}{|l|}{ Maintenance COPD Therapy Prior to Admission } \\
\hline Number of COPD Medications per Patient, mean \pm SD & $1.7 \pm 1.5$ & $1.6 \pm 1.5$ & 0.85 \\
\hline Combination LABA/ICS, n (\%) & $39(44 \%)$ & $18(38 \%)$ & 0.47 \\
\hline ICS Alone, $\mathrm{n}(\%)$ & $6(7 \%)$ & $5(10 \%)$ & 0.52 \\
\hline LAMA, n (\%) & $37(42 \%)$ & $16(33 \%)$ & 0.36 \\
\hline None, $\mathrm{n}(\%)$ & $31(35 \%)$ & $17(35 \%)$ & 0.99 \\
\hline Oxygen, n (\%) & $58(65 \%)$ & $32(65 \%)$ & 0.99 \\
\hline \multicolumn{4}{|l|}{ Comorbidities } \\
\hline None, $\mathrm{n}(\%)$ & $6(7 \%)$ & $2(4 \%)$ & 0.71 \\
\hline Chronic Kidney Disease, n (\%) & $5(7 \%)$ & $6(12 \%)$ & 0.20 \\
\hline Chronic Liver Disease, $\mathrm{n}(\%)$ & $2(2 \%)$ & $3(6 \%)$ & 0.35 \\
\hline Gastroesophageal Reflux Disease, n (\%) & $20(22 \%)$ & $9(18 \%)$ & 0.67 \\
\hline Cardiovascular Disease, $\mathrm{n}(\%)$ & $20(22 \%)$ & $17(35 \%)$ & 0.16 \\
\hline Type 1 Diabetes Mellitus, n (\%) & $1(1 \%)$ & $0(0 \%)$ & 0.99 \\
\hline Type 2 Diabetes Mellitus, n (\%) & $22(25 \%)$ & $13(27 \%)$ & 0.84 \\
\hline Heart Failure, n (\%) & $13(15 \%)$ & $6(12 \%)$ & 0.80 \\
\hline
\end{tabular}

PSI=Pneumonia Severity Index; ICU=intensive care unit; COPD=chronic obstructive pulmonary disease; ICS=inhaled corticosteroid; LABA=long-acting beta2-agonist; LAMA=long-acting muscarinic antagonist (anticholinergic)

\section{Discussion}

This is the first study to evaluate the use of systemic corticosteroids in patients with an AECOPD and CAP. Systemic corticosteroids were commonly prescribed in this patient population with $64 \%$ of patients receiving steroids. However, the use of systemic corticosteroids in this population, did not result in a shorter length of hospital stay or a lower rate of in-hospital treatment failure. Among patients with severe pneumonia, length of hospital stay was significantly longer in the steroid group compared to the non-steroids group, despite a similar mean age and PSI score. Our results are inconsistent with outcomes observed in studies of systemic corticosteroids in patients with an AECOPD. In patients with an AECOPD, systemic corticosteroids 


\section{Table 2. Outcomes According to Steroid Administration Group}

\begin{tabular}{|c|c|c|c|}
\hline & $\begin{array}{l}\text { Steroid Group } \\
\qquad(\mathrm{n}=89)\end{array}$ & $\begin{array}{l}\text { Non-Steroid } \\
\text { Group }(n=49)\end{array}$ & $p$-value \\
\hline \multicolumn{4}{|l|}{ Primary Outcome } \\
\hline Length of Hospital Stay (days), mean \pm SD & $4.7 \pm 3.2$ & $4.2 \pm 2.1$ & 0.27 \\
\hline \multicolumn{4}{|l|}{ Secondary Outcomes } \\
\hline In-Hospital Treatment Failure, ${ }^{a} \mathrm{n}(\%)$ & $6(7 \%)$ & $2(4 \%)$ & 0.72 \\
\hline ICU Admission, $\mathrm{n}(\%)$ & $4(5 \%)$ & $1(2 \%)$ & 0.66 \\
\hline Mechanical Ventilation, $\mathrm{n}(\%)$ & $1(1 \%)$ & $0(0 \%)$ & 0.99 \\
\hline Non-Invasive Ventilation, n (\%) & $2(2 \%)$ & $0(0 \%)$ & 0.54 \\
\hline Escalation of Steroid Therapy, n (\%) & $3(3 \%)$ & $1(2 \%)$ & 1.00 \\
\hline $\begin{array}{l}\text { 30-day Readmission for AECOPD or Pneumonia, } \\
\mathrm{n} / \mathrm{n}(\%)^{\mathrm{b}}\end{array}$ & $5 / 41(12 \%)$ & $2 / 30(7 \%)$ & 0.69 \\
\hline 30 day Mortality, $\mathrm{n} / \mathrm{n}(\%)^{\mathrm{b}}$ & $3 / 67(4 \%)$ & $0 / 38(0 \%)$ & 0.55 \\
\hline
\end{tabular}

an-hospital Treatment Failure: ICU admission or mechanical or non-invasive ventilation at least 6 hours after admission or after start of steroid therapy, or escalation of steroid therapy.

b30-day readmission data was available for 71 patients and 30-day mortality data was available for 105 patients.

ICU=intensive care unit; $\mathrm{AECOPD=acute}$ exacerbation of chronic obstructive pulmonary disease

\section{Table 3. Adverse Events According to Steroid Administration Group}

\begin{tabular}{|c|c|c|c|}
\hline & $\begin{array}{l}\text { Steroid Group } \\
\quad(\mathrm{n}=89)\end{array}$ & $\begin{array}{l}\text { Non-Steroid } \\
\text { Group }(n=49)\end{array}$ & $p$-value \\
\hline Insulin Required in Patients Without a History of Diabetes, $n(\%)^{a}$ & $2 / 66(3 \%)$ & $1 / 36(3 \%)$ & 0.99 \\
\hline $\begin{array}{l}\text { Hyperglycemia (>200 mg/dL) Requiring Extra Insulin Lispro in } \\
\text { Patients with a History of Diabetes, } n(\%)^{b}\end{array}$ & $11 / 23(48 \%)$ & $3 / 13(23 \%)$ & 0.18 \\
\hline $\begin{array}{l}\text { New Order for an Antipsychotic or Anxiolytic Medication for } \\
\text { Psychosis, Agitation, or Anxiety, n (\%) }\end{array}$ & $7(8 \%)$ & $7(14 \%)$ & 0.25 \\
\hline New Order for a Sedative/Hypnotic for Insomnia, n (\%) & $10(11 \%)$ & $6(12 \%)$ & 0.99 \\
\hline New Order for Loop Diuretic to Treat Edema, n (\%) & $10(11 \%)$ & $4(8 \%)$ & 0.77 \\
\hline
\end{tabular}

apercentage calculated out of number of patients in this group without diabetes

${ }^{b}$ Percentage calculated out of number of patients in this group with diabetes

\section{Table 4. Subgroup Analysis of All Patients with Severe Pneumonia ${ }^{a}$ According to Steroid Administration Group}

\begin{tabular}{|c|c|c|c|}
\hline & $\begin{array}{l}\text { Steroid Group } \\
\qquad(n=37)\end{array}$ & $\begin{array}{l}\text { Non-Steroid } \\
\text { Group }(n=25)\end{array}$ & $p$-value \\
\hline \multicolumn{4}{|l|}{ Background } \\
\hline Age in Years, mean $\pm \mathrm{SD}$ & $73.7 \pm 8.5$ & $73.7 \pm 9.9$ & 0.98 \\
\hline PSI Score, mean $\pm \mathrm{SD}$ & $111.6 \pm 14.0$ & $108.9 \pm 14.1$ & 0.47 \\
\hline \multicolumn{4}{|l|}{ Outcomes } \\
\hline Length of Hospital Stay, mean \pm SD & $6.0 \pm 4.0$ & $4.3 \pm 1.8$ & 0.03 \\
\hline In-Hospital Treatment Failure, n (\%) & $5(14 \%)$ & $2(8 \%)$ & 0.69 \\
\hline 30-day Readmission for AECOPD or Pneumonia, $\mathrm{n} / \mathrm{n}(\%)$ & $4 / 19(21 \%)$ & $1 / 16(6 \%)$ & 0.35 \\
\hline 30-day Mortality, n/n (\%) & $2 / 28(7 \%)$ & $0 / 18(0 \%)$ & 0.53 \\
\hline
\end{tabular}

asevere pneumonia: a PSI Score IV or V

PSI=Pneumonia Severity Index; AECOPD=acute exacerbation of chronic obstructive pulmonary disease 
have demonstrated reductions in treatment failure, reduced 30-day relapse, and reduced length of hospital stay. $^{9,10}$ These studies, however, excluded patients with pneumonia.

Studies examining the use of systemic corticosteroids in patients with pneumonia have included only small numbers of patients with COPD and have shown conflicting results. ${ }^{12}$ A systematic review and meta-analysis of studies examining hospitalized adults with CAP found with moderate certainty that systemic corticosteroid therapy reduced mortality by approximately $3 \%$ and the need for mechanical ventilation by approximately $5 \% .{ }^{12}$ They also demonstrated with high certainty a decreased hospital length of stay of 1 day and increased the frequency of hyperglycemia requiring treatment of $3.5 \%$. The results of this analysis were primarily driven by one large study. ${ }^{18}$ This study was a multicenter, randomized, double blind, trial of 785 patients admitted to the hospital for CAP. It demonstrated a shorter median time to clinical stability in patients who received prednisone $50 \mathrm{mg}$ daily for 7 days compared to placebo (3.0 days versus 4.4 days, $p<0.0001$ ). There was also a significantly shorter median time to effective discharge in the steroid treated group (6.0 days versus 7.0 days, $p=0.012$ ). The incidence of inhospital hyperglycemia needing insulin treatment was higher in the group that received prednisone (19\% versus $11 \%, p=0.0010)$. This study used a similar dose of prednisone and the patients had a similar severity of CAP to our study (PSI score of 86-93 versus 8688). However, only a small percentage of patients with COPD were included, $19 \%$ in the steroid group and $15 \%$ in the placebo group. There was a trend toward a larger treatment effect in patients with sepsis, who we excluded from our study. The increased incidence of hyperglycemia seen in this study is consistent with our study and others. ${ }^{12}$

Another study that showed a benefit was a randomized, controlled trial of 56 patients with severe CAP. ${ }^{19}$ There was no significant difference in the primary outcome of respiratory failure requiring mechanical or non-invasive ventilation, similar to our study. There was a significant reduction in the median time to resolution of morbidity in those patients who received corticosteroids ( 5 days versus 7 days, $p=0.02$ ). This study used a much higher dose of corticosteroid compared to our study (methylprednisolone 200mg IV bolus followed by a tapered IV dose over 9 days) and only included patients with severe CAP. While patients could have a history of COPD (4 patients in the steroid group and 2 in the non-steroid group), patients were specifically excluded if they had an AECOPD. ${ }^{19}$

While studies have shown benefits with systemic corticosteroids in CAP, ${ }^{18-21}$ several studies have showed no benefit, similar to our study. ${ }^{22-24}$ A prospective observational study by Salluh et al ${ }^{23}$ evaluated the impact of systemic corticosteroids in 111 patients with severe CAP requiring mechanical ventilation. While mortality rates were similar between patients treated with or without corticosteroids, ICU and hospital lengths of stay were significantly longer in the corticosteroid group. A quarter of the patients included in this study had a diagnosis of COPD; however, a higher percentage of patients with COPD were included in the corticosteroid group compared to the non-corticosteroid group (38\% versus $10 \%, p=0.001$ ). The median dose and duration of corticosteroids administered was $60 \mathrm{mg} /$ day methylprednisolone equivalent for 7 days. This study included patients with more severe CAP, but used a steroid dose similar to our study. A single center, prospective randomized trial compared prednisolone $40 \mathrm{mg}$ daily for 7 days to placebo in 213 patients hospitalized with CAP. ${ }^{24}$ No difference in the primary outcome of clinical cure at day 7 or 30 or in length of hospital stay was demonstrated. Analysis of patients with severe pneumonia also did not show a significant difference. A significantly higher rate of late failure ( $>72$ hours after admission) was seen in the corticosteroid group compared to the non-corticosteroid group ( $19.2 \%$ versus $6.4 \%, p=0.04)$. Approximately $20 \%$ of patients included in this study had a history of COPD. This study showed comparable results to our study. The population enrolled and the dose of corticosteroids was similar.

There are several similarities and differences between our study and the studies that evaluated systemic corticosteroids in the management of CAP. However, the biggest difference is that these studies either excluded or included very limited numbers of patients with COPD. It is possible that systemic corticosteroids do not improve outcomes in patients that have an AECOPD and pneumonia. There are a couple of hypotheses as to why this may be the case. One is the use of ICS therapy. First, patients were frequently not continued on their home COPD medication in studies of systemic corticosteroids for AECOPD. ${ }^{10}$ Therefore, we have very limited data on the true benefits of using 
systemic corticosteroids in patients continued on their home meds during hospitalization, in particular, ICS therapy. Second, several studies have shown a possible benefit of prior ICS use on outcomes in patients with COPD and pneumonia. ${ }^{25,26}$ A retrospective cohort study evaluated over 15,000 Veteran's Affairs (VA) patients with COPD hospitalized with pneumonia. ${ }^{6}$ This study found that patients with outpatient ICS use had significantly lower rates of mechanical ventilation and 30- and 90-day mortality compared to patients without outpatient ICS use. A similar study of approximately $6300 \mathrm{VA}$ patients also found a reduced mortality in patients admitted with pneumonia who were receiving an ICS for COPD. ${ }^{27}$ It is thought that ICS treatment may suppress the excessive inflammatory response that leads to harmful effects during an acute infection. ${ }^{6}$ Nearly half of the patients in our study were receiving an ICS prior to admission and during their hospital stay. It is possible that treatment with an ICS reduced the inflammatory response enough to negate the additional anti-inflammatory benefits of adding a systemic corticosteroid. Another hypothesis for a lack of benefit from corticosteroids is that the inflammation in patients with an AECOPD and pneumonia may be different and less responsive to steroids. Newer data suggest that patients with an AECOPD who have high peripheral eosinophil counts (defined as $\geq 2 \%$ eosinophils out of the total leukocyte count) have a greater benefit from systemic corticosteroids. ${ }^{28-32}$ Patients who experience an AECOPD due to CAP may not have elevated levels of blood eosinophils as exacerbations with higher eosinophil counts tend to be more non-infectious. ${ }^{33}$ It is also possible that the doses needed to alter the inflammatory response due to pneumonia might be higher than what is needed for an AECOPD due to other causes. The studies that showed a positive benefit from systemic corticosteroids tended to use higher doses than what is recommended for an AECOPD and what was used in our study. ${ }^{12,19,20}$

The major strength of our study is that it is the first to evaluate the use of systemic corticosteroids in patients with an AECOPD and pneumonia. However, due to several limitations the results need to be interpreted cautiously. The greatest limitation of our study was the retrospective design. While the baseline characteristics of the groups were similar, it is impossible to account for all possible confounding variables that could have affected the results. Another significant limitation was the number of patients with evaluable 30-day readmission or 30-day mortality data. Follow-up data for many patients was not available. Data collection for this study included patients who were admitted over nearly a 4-year time span. It is possible that changing practices in the management of AECOPD or pneumonia could have impacted our results. Last, our sample size was small and our study groups were not balanced with the steroid group being much larger than the non-steroid group. It is unknown if the results would have been different if more patients had been included, especially in the non-steroid group. However, we feel that increasing the sample size to reach power would not have resulted in a statistically significant difference in favor of the steroid group.

Our data suggest that the use of systemic corticosteroids in patients with an AECOPD and pneumonia does not decrease length of hospital stay or treatment failure rates and may increase the risk for hyperglycemia. Additionally, the use of systemic corticosteroids in patients with severe pneumonia, as defined by the PSI score, significantly increased length of hospital stay. We believe that the results of this study support the need for further research into the use of systemic corticosteroid in patients with an AECOPD and pneumonia.

\section{Declaration of Interest}

The authors have nothing to disclose. 


\section{References}

1. Kochanek KD, Murphy SL, Xu JQ, Tejada-Vera B. Deaths: Final data for 2014. National vital statistics reports; 2016;65(4). https://www.cdc.gov/nchs/data/nvsr/nvsr65/nvsr65_04.pdf Published June 2016. Accessed November 2017.

2. World Health Organization (WHO)-Media Centre. Chronic obstructive pulmonary disease (COPD) fact sheet. WHO website. http://www.who.int/mediacentre/factsheets/fs315/en/. Published November 2016. Accessed July 2017.

3. Hurst JR, Vestbo J, Anzueto A, et al. Susceptibility to exacerbation in chronic obstructive pulmonary disease. $N$ Engl J Med. 2010;363(12):1128-1138.

doi: https://doi.org/10.1056/NEJMoa0909883

4. Erkan L, Uzun O, Findik S, Katar D, Sanic A, Atici AG. Role of bacteria in acute exacerbations of chronic obstructive pulmonary disease. Int J Chron Obstruct Pulmon Dis. 2008;3(3):463467.

5. Centers for Disease Control and Prevention(CDC): National Center for Health Statistics. Pneumonia, FastStats. CDC website. https://www.cdc.gov/nchs/fastats/pneumonia.htm.

Published January 2017. Accessed July 2017.

6. Chen D, Restrepo MI, Fine MJ, et al. Observational study of inhaled corticosteroids on outcomes for COPD patients with pneumonia. Am J Respir Crit Care Med. 2011;184(3):312-316. doi: https://doi.org/10.1164/rccm.201012-20700C

7. Kew KM, Seniukovich A. Inhaled steroids and risk of pneumonia for chronic obstructive pulmonary disease. Cochrane Database Syst Rev. 2014:CD010115.

doi: https://doi.org/10.1002/14651858.CD010115.pub2

8. Søgaard M, Madsen M, Løkke A, Hilberg O, Sørensen HT, Thomsen RW. Incidence and outcomes of patients hospitalized with COPD exacerbation with and without pneumonia. Int $J$ Chron Obstruct Pulmon Dis. 2016;11(1):455-465. doi: https://doi.org/10.2147/COPD.S96179

9. Vogelmeier CF, Criner GJ, Martinez FJ, et al. Global strategy for the diagnosis, management, and prevention of chronic obstructive lung Disease 2017 report. GOLD executive summary. Am J Respir Crit Care Med. 2017;195(5):557-582. doi: https://doi.org/10.1164/rccm.201701-0218PP

10. Walters JA, Tan DJ, White CJ, Gibson PG, Wood-Baker R, Walters EH. Systemic corticosteroids for acute exacerbations of chronic obstructive pulmonary disease. Cochrane Database Syst Rev. 2014:CD001288.

doi: https://doi.org/10.1002/14651858.CD001288.pub4

11. Vondracek SF, Hemstreet BA. Is there an optimal corticosteroid regimen for the management of an acute exacerbation of chronic obstructive pulmonary disease? Pharmacotherapy. 2006;26(4):522-352. doi: https://doi.org/10.1592/phco.26.4.522
12. Siemieniuk RA, Meade MO, Alonso-Coello P, et al. Corticosteroid therapy for patients hospitalized with community-acquired pneumonia: A systematic review and meta-analysis. Ann Intern Med. 2015;163(7):519-528.

doi: https://doi.org/10.7326/M15-0715

13. Batzofin BM, Weiss YG, Ledot SF. Do corticosteroids improve outcome for any critical illness? Curr Opin Anaesthesiol. 2013;26(2):164-170.

doi: https://doi.org/10.1097/ACO.0b013e32835e820e

14. Restrepo MI, Anzueto A, Torres A. Corticosteroids for severe community-acquired pneumonia: time to change clinical practice. Ann Intern Med. 2015;163(7):560-561. doi: https://doi.org/10.7326/M15-1805

15. Mager DE, Lin SX, Blum RA, Lates CD, Jusko WJ. Dose equivalency evaluation of major corticosteroids: pharmacokinetics and cell trafficking and cortisol dynamics. $J$ Clin Pharmacol. 2003;43(11):1216-1227. doi: https://doi.org/10.1177/0091270003258651

16. Polverino E, Cillóniz C, Dambrava P, et al. Systemic corticosteroids for community-acquired pneumonia: reasons for use and lack of benefit on outcome. Respirology. 2013;18(2):263271. doi: https://doi.org/10.1111/resp.12013

17. Mandell LA, Wunderink RG, Anzueto A, et al. Infectious Diseases Society of America/American Thoracic Society consensus guidelines on the management of communityacquired pneumonia in adults. Clin Infect Dis. 2007;44 (Suppl 2):S27-72. doi: https://doi.org/10.1086/511159

18. Blum CA, Nigro N, Briel M, et al. Adjunct prednisone therapy for patients with community-acquired pneumonia: a multicentre, double-blind, randomised, placebo-controlled trial. Lancet. 2015;385(9977):1511-1518.

doi: https://doi.org/10.1016/S0140-6736(14)62447-8

19. Fernández-Serrano S, Dorca J, Garcia-Vidal C, et al. Effect of corticosteroids on the clinical course of communityacquired pneumonia: a randomized controlled trial. Crit Care. 2011;15(2):R96. doi: https://doi.org/10.1186/cc10103

20. Torres A, Sibila O, Ferrer M, et al. Effect of corticosteroids on treatment failure among hospitalized patients with severe community-acquired pneumonia and high inflammatory response: a randomized clinical trial. JAMA. 2015;313(7):677686. doi: https://doi.org/10.1001/jama.2015.88

21. Meijvis SC, Hardeman H, Remmelts HH, et al. Dexamethasone and length of hospital stay in patients with community-acquired pneumonia: A randomised, double-blind, placebo-controlled trial. Lancet. 2011;377(9782):2023-2030. doi: https://doi.org/10.1016/S0140-6736(11)60607-7

22. Mikami K, Suzuki M, Kitagawa H, et al. Efficacy of corticosteroids in the treatment of community-acquired pneumonia requiring hospitalization. Lung. 2007;185(5):249-255. doi: https://doi.org/10.1007/s00408-007-9020-3 
23. Salluh JI, Soares M, Coelho LM, et al. Impact of systemic corticosteroids on the clinical course and outcomes of patients with severe community-acquired pneumonia: A cohort study. $J$ Crit Care. 2011; 26(2):193-200.

doi: https://doi.org/10.1016/j.jcrc.2010.07.014

24. Snijders D, Daniels JM, de Graaff CS, van der Werf TS, Boersma WG. Efficacy of corticosteroids in community-acquired pneumonia: a randomized double-blinded clinical trial. Am J Respir Crit Care Med. 2010;181(9):975-982.

doi: https://doi.org/10.1164/rccm.200905-08080C

25. Ferrer M, Torres A, Martínez R, et al. Inhaled corticosteroids and systemic inflammatory response in community-acquired pneumonia: A prospective clinical study. Respirology. 2014;19(6) :929-935. doi: https://doi.org/10.1111/resp.12324

26. Ferrer M, Torres A, Reyes S, et al. Inhaled corticosteroids (ICS), systemic inflammatory response and mortality in communityacquired pneumonia (CAP). Eur Resp J. 2011;38(Suppl 55):4713.

27. Malo de Molina R, Mortensen EM, Restrepo MI, Copeland LA, Pugh MJ, Anzueto A. Inhaled corticosteroid use is associated with lower mortality for subjects with COPD and hospitalised with pneumonia. Eur Respir J. 2010;36(4):751-757. doi: https://doi.org/10.1183/09031936.00077509

28. Cardenas V, Zing L, Sharma G. Peripheral blood eosinophilia, a marker for corticosteroid responsiveness in patients hospitalized with acute exacerbation of COPD (AECOPD). Chest. 2013;144(4):710A. doi: https://doi.org/10.1378/chest.1704304

29. Bafadhel M, McKenna S, Terry S, et al. Acute exacerbations of chronic obstructive pulmonary disease: identification of biologic clusters and their biomarkers. Am J Respir Crit Care Med. 2011;184(6):662-671.

doi: https://doi.org/10.1164/rccm.201104-0597OC

30. Bafadhel M, McKenna S, Terry S, et al. Blood eosinophils to direct corticosteroid treatment of exacerbations of chronic obstructive pulmonary disease: a randomized placebo-controlled trial. Am J Respir Crit Care Med. 2012;186(1):48-55. doi: https://doi.org/10.1164/rccm.201108-15530C

31. Couillard S, Larivée P, Courteau J, Vanasse A. Eosinophils in COPD exacerbations are associated with increased readmissions. Chest. 2017;151(2):366-373.

doi: https://doi.org/10.1016/j.chest.2016.10.003

32. Bafadhel M, Greening NJ, Harvey-Dunstan TC, et al. Blood eosinophils and outcomes in severe hospitalized exacerbations of COPD. Chest. 2016;150(2):320-328.

doi: https://doi.org/10.1016/j.chest.2016.01.026

33. MacDonald M, Osadnik C, Qiu M, Vasanthakumar S, King P, Bardin P. Eosinophilia is common in copd exacerbations and associated with non-infectious aetiology. Am J Resp Crit Care Med. Th2016;193:A5185. 